\title{
EL DINERO COMO MEDIO DE CAMBIO Y SU EVOLUCIÓN: ANÁLISIS DE KATSUHITO IWAI Y SU INTERPRETACIÓN DE CARL MENGER
}

\author{
josé Guillermo peláez GRAMAjO*
}

Resumen: Este ensayo presenta un análisis de la aceptabilidad y evolución del dinero en la propuesta que hace Iwai al respecto. Él desarrolla un interesante «modelo teórico de búsqueda» del dinero como medio de cambio aceptado en las transacciones de una economía descentralizada, cuyo origen se encuentra en la idea de Carl Menger, de acuerdo con la cual esta función de la moneda emerge espontáneamente. Iwai arguye que el dinero es usado como medio de cambio universal simplemente porque todos lo usan como dinero: "el mecanismo de auto sostenimiento». Tal mecanismo permite que la economía tenga tantos equilibrios como bienes duraderos. Sin embargo, Iwai dice que el dinero nunca evolucionó naturalmente, porque debió haber habido una gran simetría -que alterara lo creado en el inicio, y entonces, existe un límite fundamental en el poder de la teoría para explicar el origen del dinero. Así la historia es un asunto esencial. El propósito de este ensayo es someter estos argumentos a un análisis crítico. En primer lugar, al contrario de lo que Iwai piensa, el mecanismo de auto sostenimiento no deteriora la teoría monetaria de Menger. Éste confirma los argumentos de Menger relacionados con la relevancia de la práctica y la costumbre en el origen del dinero. Otro resultado de este estudio muestra que la propuesta de Iwai puede completar la teoría del valor de Arrow-Debreu en el marco del intercambio, porque elimina la hipótesis de un sistema de cuentas centralizado. Finalmente, un tercer resultado relevante de este trabajo es una crítica a la teoría de Iwai, porque este muestra, en contra de él, que el equilibrio monetario depende de los "fundamentales de la economía» (técnica y preferencias), para auto sostenerse como un equilibrio de Nash.

* División de Ciencias Sociales y Humanidades, Universidad Autónoma Metropolitana, Campus de Xochimilco, México.

Procesos de Mercado: Revista Europea de Economía Política

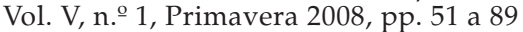


Palabras clave: Origen del dinero, dinero como medio de cambio, equilibrio monetario.

Abstract: This paper presents an analysis of the acceptability and evolution of money in Iwai's monetary proposal. He develops an interesting "search theoretical model' of money as medium that is accepted in exchange in a decentralized economy, the origin of which finds in Carl Menger idea, according to this function of money it emerges spontaneously. Iwai argues that money is used as universal medium of exchange simply because everybody uses it as money: «bootstrap mechanism». Such mechanism allows the economy to have as much monetary equilibrium as the number of durable goods in it. However, Iwai says that money never evolved naturally, because there must have been a large symmetry -breaking disturbance to create it in the beginning, and then, there is a fundamental limit on the power of the theory to explain the origin of money. History thus matters essentially. The purpose of this paper is to put these statements to a critical test. First of all, the bootstrap mechanism does not impair Menger's monetary theory, contrary to what Iwai may think. It confirms Menger's statements regarding to the practice and custom as relevant to the origin of money. Another outcome of this study shows that Iwai's proposal may complete the ArrowDebreu theory of value in the framework of exchange, because it eliminates the hypothesis of a centralized account system. Finally, a third relevant outcome of this work is a critic to Iwai theory, because it shows, against him, that monetary equilibrium depends of ureal economic» foundations (technique and preferences), to support itself as a Nash equilibrium.

Key words: Origin of money, money as medium of exchange, monetary equilibrium.

Códigos JEL: B16, E0, E42, P16.

\section{INTRODUCCIÓN}

¿Por qué se acepta la moneda a cambio de cualquier otro bien a pesar de que ésta no representa utilidad alguna para muchos agentes? Esta interrogante hace referencia a la función de medio de cambio que posee el dinero. Una referencia histórica importante es Carl Menger, quien presenta ideas interesantes sobre la negociabilidad (liquidez) de la moneda y su relación con la 
confianza. Menger argumenta que, aparentemente sería más inteligente que las personas intercambiaran mercancías buscando obtener aquéllas que generen utilidad. Se trata de una pregunta distinta a la que habitualmente se formula respecto a ipor qué los agentes desean guardar dinero en forma líquida si existen otros activos? Este es un problema de demanda que aborda la función de reserva de valor de la moneda. Al respecto la versión teórica más acabada se encuentra en los modelos de generaciones imbricadas.

Sobre la base de la «mano invisible» de Adam Smith, Menger trata de explicar la evolución del dinero. Según su propuesta, el origen del dinero lo establece un proceso social, el cual se expresa como un resultado espontáneo y no premeditado de fuerzas individuales de los miembros de la sociedad. Introduce el concepto de «vendibilidad» de un bien, según el cual una mercancía con alta vendibilidad facilita la búsqueda de los bienes necesarios a las personas poseedoras de dicha mercancía. Pero, dado que no todos los bienes poseen la misma vendibilidad, aquellos que tienen baja vendibilidad tendrán dificultades en el trueque directo. En este caso, sus poseedores buscarán intercambiar este producto con otro que tenga alta vendibilidad para después adquirir los bienes que requieren. O sea, buscarán un trueque indirecto para satisfacer sus necesidades, pues aunque el bien con alta vendibilidad no les sea útil, racionalmente lo usarán como medio de cambio en vez del trueque directo.

Esto, según Menger, se hace por interés propio, sin ningún acuerdo. Tampoco se hace por decretos legislativos relacionados con el interés público. Con el progreso de este proceso, surgen bienes aceptados por todos: el dinero como producto natural de la economía, sin intervención de ninguna autoridad. El trabajo de Iwai, en el marco de los modelos de la teoría del «search», está inspirado en la teoría monetaria de Carl Menger. La noción de «search theory» de Iwai, trata de darle rigor formal a la idea mengeriana de vendibilidad. Iwai estima que la noción de vendibilidad socava la mano invisible que Menger considera en la evolución del dinero ya que, mediante un lema (anti-Menger), Iwai muestra que la vendibilidad antes que la causa, es un efecto del origen del dinero. 
En efecto, los resultados de Iwai establecen que la naturaleza inherente del proceso descentralizado de la combinación de esfuerzos de búsqueda individual, lo que Menger denominó vendibilidad de los bienes, no constituye una característica del bien en sí, sino una variable endógena, cuya magnitud es determinada por la estructura de intercambio de la economía. El dinero es dinero porque se usa como dinero, simplemente es el producto de un mecanismo «bootstrap», pues la moneda es capaz de sostenerse por sí misma. La vendibilidad, supuesto origen de la mone$\mathrm{da}$, puede ser un efecto de ésta. Por consiguiente, para Iwai el origen del dinero no puede ser determinado únicamente por consideraciones de carácter teórico.

Este artículo muestra que el principal resultado de Iwai, el mecanismo «bootstrap», no desvirtúa sino por el contrario confirma a Menger, pues este último, al analizar el origen del dinero, también confiere gran relevancia a la práctica y la costumbre en el uso de una mercancía como moneda. En ese sentido el trabajo de Iwai puede ser interpretado como un modelo formalizado de la teoría monetaria de Menger. Un segundo resultado relevante de este estudio consiste en mostrar que la propuesta de Iwai completa la teoría del valor del equilibrio general ArrowDebreu en el marco del intercambio, pues elimina la hipótesis de un sistema de cuentas centralizado al permitir que los individuos entren en contacto cuando efectúan sus transacciones. Un tercer resultado de esta investigación constituye una crítica del modelo de Iwai, ya que éste afirma que el equilibrio monetario que formula no depende de los fundamentales de la economía. Aquí se muestra que la evolución dinámica de los mercados permite visualizar que el equilibrio monetario del modelo de Iwai sí depende de los fundamentales de la economía.

El presente trabajo se resume en cuatro secciones básicas, a saber: análisis de la teoría monetaria y del intercambio de Carl Menger, análisis de la propuesta que hace Iwai respecto a la moneda como medio de cambio, confrontación de los resultados de Iwai con la teoría de Menger, y un balance de la propuesta de Iwai. 
II

\section{LA ECONOMÍA POLÍTICA DE CARL MENGER}

\section{Teoría de la mercancía y vendibilidad}

Lo que caracteriza una economía en estado de autarquía para Menger es la autosuficiencia, en el sentido de que las actividades laborales se orientan hacia la satisfacción de necesidades, y existe una total ausencia de mercancías destinadas al intercambio. Evidentemente, esto constituye un obstáculo para la división del trabajo.

«El primer paso hacia evolución cultural económica ... se produce cuando unas personas dotadas de especiales habilidades artesanales ofrecen sus servicios a la colectividad y transforman, contra una prestación, la materia prima que se les ofrece ... El segundo paso en el camino de la evolución cultural económica, signo a la vez de creciente bienestar, se da cuando es el mismo artesano el que se procura las materias primas de sus productos, aunque estos solo los realiza por encargo expreso de los consumidores ... Este método de proveer de bienes a la sociedad significa un notable progreso tanto para los consumidores como para los productores, ya que hace que el progreso total sea más cómodo y económico, pero todavía adolece de algunos importantes inconvenientes ... Esta poco favorable situación ha hecho que los productores creen bienes de incierta venta, que conservan en sus almacenes, para poder atender al instante a una eventual demanda. Este método de atender a las necesidades de la sociedad ha llevado, en los estados más evolucionados de la economía ... de un lado a la fabricación industrial ... y del otro a la compra por parte del consumidor de mercancías ... ya acabadas ... el productor puede aprovechar al máximo la división del trabajo y el potencial de sus máquinas, con alto nivel de ahorro económico, y el consumidor alcanza la máxima seguridad ... y comodidad» 1 .

\footnotetext{
1 Menger [1871], pp. 206-207.
} 
Estos productos que productores o intermediarios poseen para el intercambio se denominan mercancías, es decir, aquellos bienes económicos susceptibles de intercambio. Por esta razón Menger manifiesta:

«...que el carácter de mercancía no es una propiedad del bien en cuestión, sino una especial relación de la misma hacia aquellas personas que disponen de ella»².

Al desaparecer esta relación, también desaparece el carácter de mercancía, cuando un individuo que dispone de la misma no intenta venderla, o bien cuando llega a manos de alguien que la consume. Además de que el carácter de mercancía no es inherente a los bienes, normalmente esta relación es pasajera. Durante el tiempo que los bienes circulan a través del intercambio tienen el carácter de mercancías. Una vez que alcanzan su objetivo económico, cuando llegan a manos del consumidor, pierden $\mathrm{su}$ «valor de cambio» y se convierten en «bienes de uso» que tienen un sentido contrapuesto al de mercancías. Con el oro, la plata, metales acuñados, etc. esto ocurre con poca frecuencia, pues raras veces desaparece la relación referida por Menger.

Una idea clave en la teoría del intercambio y en la teoría monetaria de Menger, que puede traducirse en términos de la propiedad de liquidez de un bien, es como lo señala este autor, las diferencias evidentes en la capacidad de venta («vendibilidad») de las mercancías, lo cual constituye un tema teórico muy importante. En efecto, la naturaleza y las causas de este fenómeno además de la relevancia que reviste para el éxito económico de los productos y comerciantes, influyen en la teoría del origen del dinero de Menger.

Menger argumenta que la capacidad de venta de las mercancías tropieza con limitaciones en razón del número de personas demandantes, y de la existencia de fronteras espaciales, temporales y/o cuantitativas.

2 Idem, p. 221. 


\section{Naturaleza y origen del dinero}

De acuerdo con Menger, cuando la humanidad empezó a tener conocimiento de las ventajas económicas del intercambio, sus objetivos evidentemente eran inmediatos: los individuos sólo tenían en cuenta, para sus transacciones, el valor de uso de los bienes. Todos los intercambios se limitaban a situaciones en las que

«...los bienes de que disponía un sujeto económico tenían para él menor valor de uso que los que poseía otro sujeto, mientras que para este segundo ocurría lo contrario» ${ }^{3}$.

Es natural que en estas condiciones el comercio tenía que ser muy limitado. En efecto, pocas veces un individuo posee un bien con menor valor de uso que el bien poseído por otro, y justamente este segundo opina lo contrario; además, la probabilidad de que se encuentren es todavía más reducida. Esta dificultad casi infranqueable, constituye para Menger un impedimento para la evolución de la división del trabajo y particularmente para la producción de bienes destinados a una venta incierta, el cual resuelve invocando la mano invisible de Adam Smith. En efecto, él señala que la misma naturaleza de las cosas aporta un mecanismo, gracias al cual los agentes económicos se coordinan, sin necesidad de un acuerdo especial entre ellos y menos aún una imposición del Estado. ${ }^{4}$

En las transacciones que se efectúan el objetivo económico final de los agentes es la satisfacción de sus necesidades; ésta es la razón para el intercambio por bienes que tienen para ellos mayor valor de uso. Si actuaran de manera antieconómica lo normal sería renunciar al intercambio, o efectuarlo con graves pérdidas de tiempo en busca de recibir por sus mercancías justamente los bienes requeridos y ningún otro, aunque se trate de productos que, además de ser mercancías, tienen

\footnotetext{
3 Menger [1871], p. 226.

4 Ibídem, p. 227.
} 
mayor liquidez (mayor capacidad de venta), i.e., facilitan el hallazgo de personas que ofrecen los bienes de uso y consumo requeridos. ${ }^{5}$

Lo anterior ocurre, según Menger, sin previos acuerdos, sin presión legislativa y sin atender el interés público. Menger introduce la idea de que esto sucede «bajo el poderoso influjo de la costumbre ${ }^{6}$, al aumentar la cultura económica, con una estructura tal que ciertos bienes, en razón de tiempo y lugar, poseen más capacidad de venta. ${ }^{7}$

Este proceso descrito por Menger, permite entender la gran trascendencia de la costumbre en el origen del dinero. El intercambio por mercancías con mayor capacidad de venta presupone el conocimiento de este interés en aceptar un bien, posiblemente sin utilidad alguna, a cambio de mercancías producidas con mucho esfuerzo, sólo porque tiene mayor capacidad de venta. Este conocimiento no se genera al mismo tiempo en toda la comunidad.

En estas circunstancias se advierte un origen del todo natural del dinero, pues no se trata de un invento del Estado ni de un producto legislativo.

Como puede verse, Menger ofrece dos pistas para entender la naturaleza y el origen del dinero. Por una parte la idea de «vendibilidad» de las mercancías, según la cual, aquellas con mayor capacidad de venta se van imponiendo como medio de cambio cuando el comercio se va extendiendo en la sociedad en un contexto de incertidumbre. Esta producción de mercancías destinada a una venta incierta permite la evolución de la división social del trabajo, pues en un inicio son algunos individuos más hábiles los que se percatan de las ventajas de utilizar como medio de cambio las mercancías con mayor vendibilidad. Esta heterogeneidad social respecto al aprovechamiento de la rentabilidad que el intercambio de mercancías ofrece, va desapareciendo a medida que el comercio se expande a toda la sociedad

\footnotetext{
5 Ibídem, pp. 227-228.

6 Ibídem.

7 «A estos bienes llamaron los germanos Geld (dinero),...en alemán dinero significa,...objeto que vale, que sirve para pagar». Ibídem.
} 
y la moneda mercancía termina por imponerse como medio de cambio bajo el poderoso influjo de la costumbre, lo cual constituye la segunda pista que Menger formula en su teoría sobre la naturaleza y origen del dinero.

\section{III \\ MODELO BÁSICO DE INTERCAMBIO \\ DESCENTRALIZADO DE IWAI}

\section{Datos y supuestos básicos}

Se trata de una economía con un gran número de agentes que tienen vida infinita y $n$ bienes de consumo $i=1 \ldots n$, indivisibles. Cada agente produce una unidad de un único bien y consume una unidad de otro único bien. Por consiguiente, cada miembro de esta economía se clasifica por el par ordenado de índices $(i, j)$, siendo i el bien que es capaz de producir y j el bien que tiene necesidad de consumir. Se excluye la existencia de individuos que correspondan al par ordenado $(\mathrm{i}, \mathrm{i})$. La frecuencia de población productora de i y consumidora de j se denota por $e_{i, j^{\prime}}$ y se denomina frecuencia de habilidad necesidad. Por hipótesis (división social del trabajo) se tiene por lo tanto $e_{i, i}=0$. De todo lo anterior se infiere:

$$
\sum_{i} \sum_{j} e_{i, j}=1
$$

Entonces, toda la estructura «real» de esta economía se resume en las frecuencias de habilidad-necesidad, $\left\{e_{i, j}\right\}$, que expresan la forma como Iwai concibe que la tecnología y las preferencias están distribuidas entre los agentes económicos. Iwai distingue cuatro tipos posibles de distribución de la tecnología y las preferencias entre los agentes, los cuales se describen en la siguiente gráfica, para $n=4$. Si existe un número positivo de agentes que producen 2 y consumen 3 , se dibuja una flecha de 2 a 3 . Si ningún productor de 1 consume 3 , no habrá flecha de 1 a 3 . El grosor de la flecha expresa la frecuencia. 


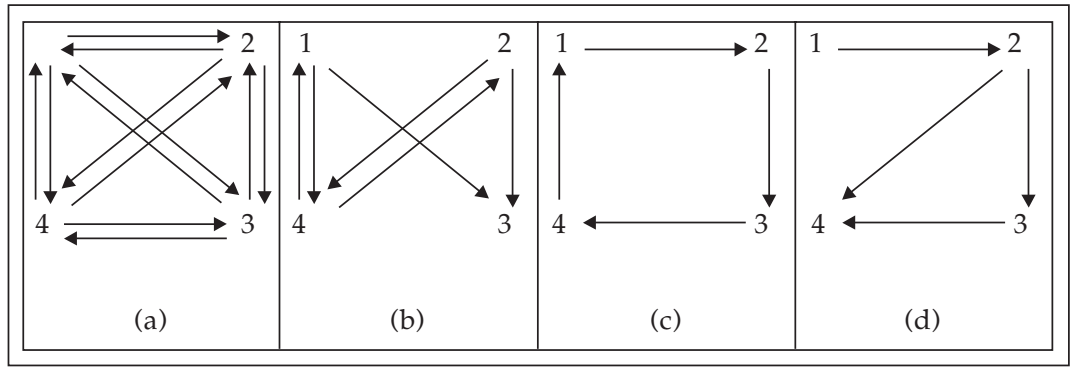

Si existe un número positivo de agentes que producen dos. En la Gráfica anterior, a) es una economía plenamente conecta$\mathrm{da} ; \mathrm{b}$ ) es una economía menos que plenamente conectada pero más que mínimamente conectada, c) es una economía mínimamente conectada y d) es una «economía» desconectada.

\section{Modelo de intercambio descentralizado}

La importancia de la conexión en este modelo, radica en el hecho de ser el requisito mínimo para que una asociación de agentes sea denominada una economía. En la medida que una economía está conectada, en principio todos sus miembros pueden satisfacer sus necesidades y superar el estado de autarquía mediante un intercambio descentralizado. En tanto que, en una economía desconectada no es posible ninguna coordinación.

Ahora Iwai formula el análisis de dos interrogantes, a saber:

1) ¿Es posible que una forma descentralizada de las decisiones de los individuos permita una coordinación del intercambio que elimine la autarquía, i.e., que sin el soporte de una autoridad central emerja una economía conectada?

2) Si esto es posible ¿es capaz, tal coordinación descentralizada, de evolucionar hacia una economía monetaria en forma espontánea a partir de una estructura económica «real» históricamente dada, i.e., sin la intervención de una autoridad externa?

La primera pregunta tiene que ver con la existencia y la segunda con la evolución de un sistema de intercambio descentralizado. 
Para el autor es de suma importancia separar estas dos interrogantes, al menos conceptualmente. Iwai responde estas preguntas desarrollando un modelo de economía de intercambio descentralizado. Es un modelo inscrito dentro de los recientes trabajos de investigación teórica sobre moneda («search theoretic») ${ }^{8}$. A este efecto divide la economía en un número de zonas de intercambio separadas, cada una de las cuales se especializa en intercambios entre un par de bienes dado, y le permite a la gente elegir la zona de intercambio a visitar. Además supone que en una zona de intercambio los encuentros entre los agentes se efectúan aleatoriamente. Por lo tanto en esta economía existen $\left(\begin{array}{l}n \\ 2\end{array}\right)=\frac{n(n-1)}{2}$ zonas de intercambio ${ }^{9}$. Se denota como $q_{i, j}$ a la frecuencia de la oferta de $i$ y demanda de $j, q_{i, i}=0$ por hipótesis $\mathrm{y}$

$$
\sum_{i} \sum_{j} q_{i, j} \leq 1
$$

El conjunto frecuencia oferta-demanda $\left\{q_{i, j}\right\}$, que es el principal determinante de la conducta individual de búsqueda de los miembros de la economía, en general difiere, excepto en el intercambio de trueque, del conjunto frecuencia habilidad-necesidad $\left\{e_{i, j}\right\}$, que representa los fundamentales de la economía.

Si cada zona de intercambio es grande, el proceso que gobierna los encuentros de los agentes puede ser razonablemente representado por un proceso de Poisson ${ }^{10}$. Así, el modelo asume que la probabilidad de que un i oferente, $\mathrm{j}$ demandante, encuentre un j oferente, i demandante, en la zona $(i, j)$ durante una intervalo de tiempo muy corto, es proporcional a $q_{j, i}$, es decir, a la frecuencia del último (probabilidad de frecuencia relativa). Dado que se tiene un grado de libertad para elegir la unidad de tiempo,

${ }^{8}$ Iwai [1988a], [1988b], [1989] y [1997].

9 Similar al número de mercados del modelo de equilibrio general antes del numerario.

${ }^{10}$ Ver anexo al respecto; $\mathrm{q}_{\mathrm{i}, \mathrm{j}}$ representa un parámetro probabilístico del modelo. 
se puede establecer esta probabilidad igual a $q_{j, i}$ por unidad de tiempo en el proceso de Poisson.

Así, la frecuencia de oferta y demanda $q_{j, i}$, permite al autor formalizar lo que Menger denominó «vendibilidad» de bienes. La posesión de cierto bien, i por ejemplo, con alta $q_{j, i}$ podría facilitar a un agente la búsqueda individual de otro agente que quiere ofrecer el bien j que el demanda, reduciendo así sus costos de búsqueda. Asimismo, el modelo interpreta que un bien con alta $q_{i, j}$, tiene alta «comprabilidad», aunque el mismo Menger no usó esta noción. Esto debido a que su posesión facilita la búsqueda individual de otro individuo que demanda el bien i que él ofrece, reduciendo así el coste de búsqueda.

\section{Estrategia individual}

Ante la ausencia de una autoridad central que coordine los intercambios de bienes de consumo, los individuos deben buscar por sí mismos patrones de intercambio para obtener los bienes que necesitan. Considérese el i productor, $\mathrm{j}$ consumidor, que ha completado su producción y posee una unidad de i en sus manos. Se abre un abanico de estrategias alternativas. Una posibilidad es una estrategia autárquica. También puede acudir directamente a la zona de intercambio $(i, j)$ en busca de alguien que desee intercambiar j por i. Se asume que la tasa de intercambio es uno por uno, sin encarar en este modelo el problema de la formación de los precios.

También puede acudir primero a la zona $(\mathrm{i}, \mathrm{k})$, en busca de otro agente que desee intercambiar k por i. Luego, presentarse en la zona $(k, j)$ y buscar otro agente que desee intercambiar $\mathrm{j}$ por $\mathrm{k}$. Esta estrategia de «intercambio indirecto» utiliza el bien k como «medio de cambio». Las estrategias de intercambio indirecto que utilizan más de un medio de cambio también son posibles.

Una vez que el i productor, j consumidor, obtiene el bien j, cualquiera que sea la estrategia de intercambio que use para el efecto, retorna a casa y consume $\mathrm{j}$ a fin de prepararse para una nueva producción de i. Este ciclo, al margen de la autarquía, de producción - búsqueda - intercambio - consumo, se repite una y otra vez. 


\section{Programa de búsqueda óptima}

El modelo supone que el i productor, $\mathrm{j}$ consumidor, vive siempre y descuenta las utilidades futuras mediante una tasa de descuento de tiempo $r>0$. Recibe una utilidad $u>0$ por consumir una unidad de $j$, pero ninguna utilidad de ningún otro bien, incluyendo su propio producto. Para simplificar el análisis, Iwai asume que no se incurre en tiempo ni en costes en la producción, en el intercambio y en el consumo. La introducción de estos dos factores es sumamente fácil. También se asume por simplicidad analítica, que las expectativas de este agente sobre los parámetros futuros son estacionarias ${ }^{11}$. Este último supuesto permite trabajar únicamente con programas de estado estacionario. Evidentemente, este supuesto es bastante restrictivo, en particular para el estudio de la evolución del dinero. Una consecuencia trivial de este supuesto es que la utilidad esperada del tiempo de vida de permanecer en autarquía es cero. Por consiguiente, para poner en acción al agente productor-consumidor, el modelo debe garantizar una utilidad esperada estrictamente positiva.

Si él ha participado en un intercambio de trueque, $V_{i \phi j}$ denota la utilidad esperada del tiempo de vivido descontado para el agente i productor, $\mathrm{j}$ consumidor, con su producto en la mano. Entonces, por programación dinámica, puede calcularse su valor. Al considerar un pequeño intervalo de tiempo $\Delta>0$, por proceso de Poisson $q_{j i} \Delta^{12}$ denota la probabilidad de que el productor-consumidor encuentre uno de sus socios de trueque durante dicho intervalo. En este caso recibe la utilidad $u$ por consumir $j$, produce una nueva unidad de $i, y$ luego inicia otra vez una actividad de búsqueda de $\mathrm{j}$ con la misma utilidad esperada. La probabilidad de que no lo encuentre se denota por $1-q_{j i} \Delta$. Si este es el caso, no aumenta su utilidad, excepto que ha gastado el tiempo $\Delta$.

${ }^{11}$ Lo cual significa que el valor corriente hoy, cualquiera que éste sea, regirá en el futuro.

12 Véase en anexo $f(1)$, donde el parámetro $\mathrm{q}_{\mathrm{i}, \mathrm{j}}$ es reemplazado por $\lambda$. 
La utilidad total descontada si lo encuentra se denota por $\frac{u+V_{i \phi j}}{1+r \Delta}$, en tanto que $\frac{V_{i \phi j}}{1+r \Delta}$ denota la utilidad total descontada si no lo encuentra. Por consiguiente, se tiene la esperanza matemática

$$
V_{i \phi j}=\frac{q_{j i} \Delta\left(u+V_{i \phi j}\right)+\left(1-q_{j i} \Delta\right) V_{i \phi j}}{1+\Delta r}=\frac{u q_{j i}}{r}=\frac{u}{\left(1+\frac{r}{q_{j i}}\right)-1}
$$

Ahora, se denota por $V_{i k j}$ la utilidad esperada del tiempo vivido descontado con $\mathrm{k}$ como único medio de cambio, del agente i productor, j consumidor. Para efectos de cálculo, $V_{i k j}^{\prime}$ denota la utilidad esperada del tiempo vivido cuando tiene dinero (mercancía k) en sus manos. Luego por método de programación dinámica se relacionan estas dos utilidades de tiempo vivido durante un $\Delta>0$ así:

$$
\begin{gathered}
V_{i k j}=\frac{q_{k i} \Delta V_{i k j}^{\prime}+\left(1-q_{k i} \Delta\right) V_{i k j}}{1+r \Delta} \\
V_{i k j}^{\prime}=\frac{q_{j k} \Delta\left(u+V_{i k j}\right)+\left(1-q_{k j} \Delta\right) V_{i k j}^{\prime}}{1+r \Delta}
\end{gathered}
$$

Al reagrupar términos y cancelando $\Delta$, se tiene:

$$
V_{i k j}=\frac{u}{\left(1+\frac{r}{q_{k i}}\right)\left(1+\frac{r}{q_{j k}}\right)-1} \forall k \neq i, j
$$

Por el mismo procedimiento $V_{i k l \ldots g h j}$, denota la utilidad esperada de descuento del agente i productor, $\mathrm{j}$ consumidor, con su producto en la mano, cuando ha participado en intercambios indirectos que usa $\mathrm{k}, \mathrm{l}, \ldots, \mathrm{g}, \mathrm{h}$ como medios de cambio. Aplicando el mismo método de programación dinámica se tiene: 


$$
V_{i k l \ldots g h j}=\frac{u}{\left(1+\frac{r}{q_{k i}}\right)\left(1+\frac{r}{q_{l k}}\right) \ldots\left(1+\frac{r}{q_{h g}}\right)\left(1+\frac{r}{q_{j h}}\right)-1}
$$

Es obvio que la estrategia óptima de búsqueda del agente i productor, $\mathrm{j}$ consumidor, es aquélla que maximiza el valor de las expresiones anteriores. La regla de desempate de dos estrategias con la misma utilidad esperada es lexicográficamente aleatoria, por lo cual elige la estrategia con la sucesión más corta de intercambios, cuando estas tienen diferente longitud, o el lanzamiento de una moneda bien balanceada, cuando tienen la misma longitud. Dado que el número de posibles estrategias es esencialmente finito, entonces siempre existe un programa óptimo. No obstante, si la utilidad máxima es nula, la estrategia óptima es la autarquía.

Nótese que todas las expresiones anteriores son de la forma $\frac{u}{L-1}$. En consecuencia, el programa óptimo de búsqueda puede reformularse de manera más consistente mediante el siguiente lema:

\section{Lema 1}

El programa óptimo de búsqueda para un i productor, $\mathrm{j}$ consumidor, es elegir un conjunto de índices, $k, 1, \ldots, g$, h que minimiza la siguiente suma:

$$
\log \left(1+\frac{r}{q_{k i}}\right)+\log \left(1+\frac{r}{q_{l k}}\right)+\ldots+\log \left(1+\frac{r}{q_{h g}}\right)+\log \left(1+\frac{r}{q_{j h}}\right)
$$

Si el valor mínimo es infinito, el agente elige una estrategia de autarquía. Si este valor es nulo elige un intercambio de trueque. En cualquier otro caso, elegirá el intercambio indirecto que minimiza el conjunto de bienes $\mathrm{k}, \mathrm{l}, \ldots, \mathrm{g}$, h como medios de cambio. 


\section{Demostración}

Evidentemente, L está acotado por abajo, pues $L \geq 1 \rightarrow L-1 \geq 0$, entonces $\frac{u}{L_{\text {Min }}-1} \geq \frac{u}{L-1} \forall L, L-1 \geq L_{\text {Min }}-1 \rightarrow L \geq L_{\text {Min }}$. Puesto que $\log (L)$ es creciente, entonces también $\log (L) \geq \log \left(L_{\text {Min }}\right)$, lo cual completa la demostración pues se tiene como consecuencia que $\operatorname{Max} \frac{u}{L-1} \equiv \operatorname{Min} L$, o Min $\log (L)$.

La formulación del lema anterior tiene una interpretación importante. El $\log \left(1+\frac{r}{q_{k, l}}\right)$ es una función decreciente de $q_{k, l}$, con un valor que tiende a infinito a medida que $q_{k, l}$ tiende a cero. Su valor también es creciente a medida que el valor de la tasa de descuento $r$ se incrementa. Por consiguiente, este valor puede ser considerado como un índice del coste de oportunidad de búsqueda para el agente k oferente, 1 demandante, en la zona de intercambio $(\mathrm{l}, \mathrm{k})$. Ante esta interpretación, el productor consumidor con vida infinita puede determinar su programa óptimo de búsqueda sumando los costes de búsqueda en cada zona de intercambio y minimizando su suma. Esto es como si tuviera un tiempo de vida finito ${ }^{13}$ y no existiera tiempo de descuento. En lo que continúa Iwai explota esta simplificación.

\section{El Sistema de intercambio descentralizado como un equilibrio de Nash}

Iwai ha mostrado que existen varios sistemas de intercambio que pueden ser utilizados para el estudio del origen y la naturaleza del dinero. Aquí hemos analizado dos: el sistema de intercambio de trueque y el sistema de intercambio de mercancía moneda ${ }^{14}$.

\footnotetext{
13 Esto implica una simple reinterpretación de los símbolos del Lema 1.

14 También el sistema de intercambio con moneda fiduciaria, así como el sistema de intercambio de regalos bajo un enfoque antropológico. Estos sistemas no los
} 
Puesto que uno de los problemas que debe resolver el modelo de Iwai es analizar la posibilidad de intercambios que eliminen la autarquía sin la coordinación de una autoridad centralizada, entonces, ahora el autor formula este problema de una manera analítica más manejable con la siguiente interrogante: ¿es posible, y bajo qué condiciones, que cualquiera de los sistemas de intercambio descentralizados arriba mencionados pueda ser sostenido como un tipo de equilibrio no cooperativo? La noción de equilibrio no cooperativo que Iwai usa es el equilibrio de Nash ${ }^{15}$. Por simplicidad, Iwai sólo estudia el equilibrio de Nash de estado estacionario.

Aunque el equilibrio de autarquía es el peor escenario posible ya que, excluye todo sistema de intercambio, a continuación se formula una proposición que constituye la base de las conclusiones de Iwai, pues se utiliza para establecer el precepto del equilibrio monetario y del mecanismo de origen del dinero.

\section{Proposición}

Una autarquía completa es un equilibrio de Nash en cualquier economía.

\section{Demostración}

Supóngase que en cualquier zona de intercambio no aparece nadie, de modo tal que cualquier $q_{i, j}$ es cero. Entonces, por la ecuación (8), el costo total de búsqueda de cualquier estrategia de intercambio es infinito. Por consiguiente, todos eligen estar en autarquía, y toda $q_{i, j}$ viene a ser nula, tal como se supuso, lo cual completa la demostración.

examinaremos en este trabajo, puesto que para los fines de este artículo, el estudio de la naturaleza y origen del dinero, alcanza con el sistema de moneda mercancía.

15 Un equilibrio de Nash es un perfil de estrategias tal que, en lo concerniente a cada individuo, su estrategia es tan buena respecto a lo que se supone que harán los restantes agentes, como cualquier otra estrategia disponible para él. 
La demostración anterior, aunque trivial, permite que Iwai establezca el esqueleto de la estructura general del equilibrio de Nash en la economía de intercambio descentralizada. Un conjunto de frecuencias de oferta-demanda, $\left\{q_{i, j}\right\}$, determina las acciones óptimas de todo miembro de la economía, cuya agregación establece el valor corriente de dicho conjunto. Esto implica una causalidad circular, y si existe este conjunto $\left\{q_{i, j}\right\}$ que sea consistente con dicha causalidad, esto constituye un equilibrio del modelo.

La circularidad inherente a la vendibilidad revela una disociación de causa y efecto de ésta. En un primer momento, el valor nulo de la vendibilidad, i.e., costes infinitos de transacción, determina una conducta autárquica de cada uno de los agentes. Luego, en un segundo momento, esta conducta autárquica de todos los agentes trae como consecuencia un conjunto de ofertas y demandas nulas en todos los mercados, i.e., una vendibilidad nula para todas las mercancías. Esta circularidad de la vendibilidad de las mercancías constituye la piedra angular del razonamiento de Iwai para la generación de su principal resultado en relación con el origen del dinero, como se verá a continuación.

\section{IV \\ LA NATURALEZA DEL AUTOSOSTENIMIENTO (BOOTSTRAP) DEL SISTEMA DE INTERCAMBIO DE MERCANCÍA DINERO ${ }^{16}$}

En el sistema con mercancía dinero, Iwai indexa este bien como m. El primer problema del modelo es determinar bajo que condiciones todos los integrantes de la economía voluntariamente llegan a usar este bien como el único medio de cambio, aun si esto requiere que incurran en costes de búsqueda no una sino dos veces. Esto exceptúa, por supuesto, a aquéllos que producen o consumen dicho bien. A tal efecto, Iwai formula el siguiente lema.

16 El sistema de intercambio de trueque no se examina, pues es sabido que en el mismo se bloquean los intercambios. Un análisis completo de este problema puede verse en Benetti [1990] pp. 23-38. 


\section{Lema 2}

Un bien $m$ es usado como el único medio de cambio por todos los miembros de la economía (excepto si es productor o consumidor), si el conjunto de frecuencias demanda-oferta $\left\{q_{i j}\right\}$ que el agente observa en las zonas de intercambio satisface el siguiente conjunto de desigualdades para todo i y para todo j:

$$
\begin{gathered}
\log \left(1+\frac{r}{q_{m i}}\right)+\log \left(1+\frac{r}{q_{j m}}\right)<\infty \\
\log \left(1+\frac{r}{q_{m i}}\right)+\log \left(1+\frac{r}{q_{j m}}\right)<\log \left(1+\frac{r}{q_{j i}}\right) \\
\log \left(1+\frac{r}{q_{m i}}\right)+\log \left(1+\frac{r}{q_{j m}}\right)<\log \left(1+\frac{r}{q_{k i}}\right)+\log \left(1+\frac{r}{q_{j k}}\right) \forall k \neq m \\
\log \left(1+\frac{r}{q_{j m}}\right) \leq \log \left(1+\frac{r}{q_{k m}}\right)+\log \left(1+\frac{r}{q_{j k}}\right) \forall k \neq m \\
\log \left(1+\frac{r}{q_{m i}}\right)<\infty \\
\log \left(1+\frac{r}{q_{m i}}\right) \leq \log \left(1+\frac{r}{q_{m k}}\right)+\log \left(1+\frac{r}{q_{k i}}\right) \forall k \neq m
\end{gathered}
$$

Las desigualdades (9), (10) y (11) establecen, respectivamente, que es menos costoso usar m como medio de cambio que estar 
en autarquía, que intercambiar directamente, y que usar cualquier otro bien como medio de cambio.

Las desigualdades (12) y (13) están relacionadas con la conducta de dos grupos de individuos: los que están en su segunda búsqueda de bienes consumibles y los afortunados que han producido el bien monetizado $\mathrm{m}$. Dichas desigualdades establecen, respectivamente, que cuando un individuo posee el bien $\mathrm{m}$ (ya sea por previo intercambio o por producción) es menos costoso intercambiarlo directamente por su bien consumible que estar en autarquía y que usar algún otro bien como medio de cambio.

Las desigualdades (14) y (15) se relacionan con la conducta de otro afortunado agente que consume el bien monetizado $\mathrm{m}$. Estas dicen, respectivamente, que cuando se tiene la necesidad de consumir el bien m es menos costoso buscar un trueque directo que estar en autarquía y que usar algún otro bien como medio de cambio ${ }^{17}$.

Este lema confirma la lógica de la teoría monetaria de Menger en el marco del modelo teórico de búsqueda. Un bien m es usado como moneda si y solamente si su vendibilidad $q_{i m}$ y su comprabilidad $q_{m j}$ son uniformemente mayores que la de todos los otros bienes de la economía, $q_{i j}(i, j \neq m)$. Por consiguiente, también se verifica la primera mitad de la causalidad circular que establece el equilibrio de Nash. Sin embargo, de acuerdo con Iwai, esta lógica mengeriana se colapsa si la circularidad metodológica que él formula se lleva hasta sus últimas consecuencias.

El análisis de la posibilidad de que este sistema de intercambio con moneda mercancía se sostenga como un equilibrio de Nash, requiere examinar la otra mitad de la causalidad. Es decir, la determinación de los valores de las frecuencias ofertademanda (vendibilidad-comprabilidad) $\left\{q_{i, j}\right\}$, como resultado de la agregación de las actividades individuales de búsqueda.

Ahora Iwai invoca a Robert Clower, al sostener que «el dinero compra bienes, los bienes compran dinero, pero los bienes no compran bienes». Esta proposición es trasladada a la determinación del conjunto $\left\{q_{i, j}\right\}$, en un sistema de intercambio.

\footnotetext{
17 La demostración de este lema puede verse en Iwai [1977].
} 
Primero, el hecho de que «bienes no compran bienes» implica que en un sistema de intercambio mercancía dinero, nadie busca intercambiar bienes no monetarios por trueque directo. De modo que la zona de intercambio para todo par de bienes no monetarios, i.e., $j \neq m$, es totalmente vacía, lo cual implica que:

$$
q_{i j}=0 \forall i, j \neq m
$$

Luego, si «los bienes compran dinero» $\mathrm{y}$ «el dinero compra bienes» significa que, en un sistema de intercambio con mercancía moneda, todo agente que no produce ni consume el bien monetizado $\mathrm{m}$, primero busca intercambiar su producto $i \neq m$ por el bien monetizado $\mathrm{m}$, para después buscar intercambiar el bien monetizado $\mathrm{m}$ por su bien consumible $j \neq m$. O sea que la gente primero vende su producto (compra dinero de acuerdo con Clower), y luego compra lo que consume.

Esto significa que un i productor, $\mathrm{j}$ consumidor, la primera mitad de su actividad la invierte en la zona de intercambio $(\mathrm{i}, \mathrm{m})$, y la segunda mitad en la zona de intercambio $(m, j)$. Por consiguiente, $q_{m i}$ denota la probabilidad, por unidad de tiempo, de encontrarse con un comprador de su producto i en la zona $(\mathrm{i}, \mathrm{m})$. Mientras que $q_{j m}$ expresa la probabilidad, por unidad de tiempo, de encontrar un vendedor de su producto consumible en la zona de intercambio $(\mathrm{m}, \mathrm{j})$. Por proceso de Poisson, $\frac{1}{q_{m i}} 18$ es la expectativa de tiempo de búsqueda en la primera zona de intercambio (tiempo de venta), y $\frac{1}{q_{j m}}$ constituye la expectativa de tiempo de búsqueda en la segunda zona (tiempo de compra). Este agente repite este ciclo de venta-compra una y otra vez.

$$
\text { Ante estos hechos, } \frac{\frac{1}{q_{m i}}}{\frac{1}{q_{m i}}+\frac{1}{q_{j m}}}=\frac{q_{j m}}{q_{m i}+q_{j m}} \text { representa la fracción }
$$

de tiempo vivido gastado en la primera zona como vendedor de i.

18 Véase el anexo. En la parte final del mismo se obtiene este resultado con $\mathrm{q}_{\mathrm{i}, \mathrm{j}}=\lambda$. 
A la vez, $\frac{\frac{1}{q_{j m}}}{\frac{1}{q_{m i}}+\frac{1}{q_{j m}}}=\frac{q_{m i}}{q_{m i}+q_{j m}}$, muestra la fracción de tiempo vivido gastado en la segunda zona como comprador de $\mathrm{j}$.

Si ahora se supone que la economía se encuentra en estado estable, entonces por un «silogismo» probabilístico, se tiene que estas dos fracciones de series de tiempo de un buscador individual pueden identificarse como evento seguro (probabilidad igual a uno), con las fracciones de todos los i productores, $\mathrm{j}$ consumidores, que actúan como vendedores en la zona de intercambio $(\mathrm{i}, \mathrm{m})$ y como compradores en la zona de intercambio $(\mathrm{j}, \mathrm{m})$.

Por lo tanto, sus frecuencias de estado estable en zona ( $\mathrm{i}, \mathrm{m}$ ) son $\left(\frac{\frac{1}{q_{m i}}}{\frac{1}{q_{m i}}+\frac{1}{q_{j m}}}\right) e_{i j}$ y sus frecuencias de estado estable en zona $(\mathrm{m}, \mathrm{j})$ son $\left(\frac{\frac{1}{q_{j m}}}{\frac{1}{q_{m i}}+\frac{1}{q_{j m}}}\right) e_{i j} \forall i, j \neq m$. Si se construye el agregado de estas frecuencias, sin olvidar añadir la frecuencia del consumidor de $\mathrm{m}$ y la del productor de $\mathrm{m}$, se obtiene:

$$
q_{i m}=e_{i m}+\sum_{h \neq m} \frac{\frac{1}{q_{m i}}}{\frac{1}{q_{m i}}+\frac{1}{q_{h m}}} e_{i h}
$$

que representa el valor del estado estable del sistema monetario de intercambio de bienes. El segundo término del lado derecho constituye la suma de todas las demandas sociales de $m$ como medio de cambio (bienes que compran m). Mientras que $e_{i, m}$ implica que la moneda como institución todavía requiere un mínimo 
soporte de condiciones reales de la economía. La suma de esos dos términos expresa de demanda general (vendibilidad general).

$$
q_{m i}=e_{m i}+\sum_{k \neq m} \frac{\frac{1}{q_{i m}}}{\frac{1}{q_{m k}}+\frac{1}{q_{i m}}} e_{k i}
$$

que representa el valor del estado estable del sistema monetario de intercambio de bienes. El segundo término del lado derecho constituye la suma de todas las ofertas sociales de $m$ como medio de cambio (m que compra bienes). Por su parte $e_{m, i}$, también refleja que la moneda aún necesita un mínimo de propiedades reales. La suma de esos dos términos expresa la oferta general (comprabilidad general).

Puesto que, de acuerdo con (16), esas demandas y ofertas sociales de $\mathrm{m}$ como medio de cambio no son nulas, entonces se sostienen como un equilibrio monetario por sí mismo, sin ninguna demanda y oferta real de moneda. Así, en (17) y (18) $q_{i, m}$ y $q_{m, i}$, vendibilidad y comprabilidad en estado estable de $m$ frente a bienes no monetarios respectivamente, ahora no son causa sino efecto del uso del bien monetizado $m$ como moneda. Una vez que m empieza a usarse como moneda, emerge una estructura de intercambio totalmente asimétrica en el mundo de los bienes: se eleva al máximo la vendibilidad de $m\left(q_{i, m}\right)$, al igual que su comprabilidad $\left(q_{m, i}\right)$, a expensas de las de otros bienes, pues reiterando (16): $q_{i, m}=0 \quad \forall i, j \neq m$, aun y cuando originalmente $q_{i, m}$ y $q_{m, i}$ hayan sido bajas. Esto es lo que se define como mecanismo «bootstrap»: $\mathrm{m}$ se sostiene por sí misma sin ningún soporte real.

El análisis que se ha hecho para mostrar el lema anterior, aunque pedestre tiene un carácter heurístico que describe el comportamiento del equilibrio monetario en estado estable de largo plazo, tal como lo presenta Iwai [1997]. Sin embargo, como se verá mas adelante, este análisis tiene el inconveniente de que encubre los problemas que surgen en el desequilibrio que se genera al elegir uno de los bienes como medio universal de cambio, con lo cual desaparecen la mayoría de las zonas de intercambio 
originales, pues bajo estas nuevas condiciones sólo hay n-1 mercados (todos aquéllos donde participa la mercancía m). Al final de este artículo hacemos un balance de la propuesta de Iwai; ahí hacemos un análisis de estos problemas que han quedado ocultos.

\section{Lema 3}

Si una economía conectada usa uno de sus bienes reales como único medio de cambio, entonces todas sus vendibilidades y comprabilidades son estrictamente positivas en estado estable. Si el bien monetizado es $\mathrm{m}$, se tiene:

$$
q_{i, m}>0 \text { y } q_{m, i}>0 \quad \forall i
$$

Este lema enseña que si la economía está conectada, el bien monetizado $\mathrm{m}$ es constantemente ofrecido y demandado a cambio de todos los otros bienes. Puesto que según (16) existe absoluta ausencia de vendibilidad y comprabilidad de cualquier bien no monetario en un sistema de intercambio con moneda-mercancía, entonces surge una de las principales proposiciones de Iwai.

\section{Proposición}

Toda economía conectada puede sostener un sistema de mercancía-moneda como un equilibrio de Nash.

\section{Demostración}

Si se sustituye (16) y (18) en ambos lados de las desigualdades i)-vii) del lema 2, resulta trivial satisfacer estas desigualdades. Esto significa que todo agente económico, con excepción del

\footnotetext{
19 La demostración también puede verse en Iwai [1997].
} 
productor y el consumidor del bien monetizado, utiliza voluntariamente $\mathrm{m}$ como moneda. También es evidente que la elección de $m$ es arbitraria, que era lo que se deseaba demostrar.

Un sistema de intercambio con mercancía-moneda es así capaz de sostenerse por sí mismo como equilibrio de Nash sin ningún soporte «real». Debido a que la moneda trasciende la realidad es capaz de superar las restricciones reales de la economía y hace posible el intercambio descentralizado que, de otra manera, sería imposible.

\section{$\mathrm{V}$}

\section{EVALUACIÓN DE LA PROPUESTA DE IWAI}

\section{Comparación de la teoría de Iwai con la de Menger}

a) La dificultad de una evolución natural del sistema moneda-mercancía según Iwai

Para el modelo de Iwai, un sistema de intercambio con moneda-mercancía es potencialmente un sistema ubicuo, en el sentido de que puede sostenerse como un equilibrio en cualquier economía conectada. Pero la ubicuidad «potencial» no debe confundirse con la ubicuidad «real». Es decir, el que pueda existir no significa que exista ese equilibrio. Por el contrario, el mecanismo «bootstrap» que habilita el sistema de intercambio con mercancía-moneda con su ubicuidad potencial, trabaja en contra de su evolución «natural». Su trascendencia de la realidad impide que la mayoría de las economías evolucionen de una manera espontánea.

Para ver esto, obsérvese de nuevo la Gráfica de los tipos de economía. Evidentemente, la economía desconectada no tiene oportunidad de evolucionar de forma natural a un sistema con mercancía-moneda. Pero lo importante es que tampoco las economías mínimamente conectadas y más que mínimamente conectadas, pero menos que plenamente conectadas, pueden evolucionar naturalmente hacia un sistema de intercambio con moneda mercancía. Así, por ejemplo, aun cuando en ambas economías existe al menos 
un productor del bien 2 y consumidor del bien 3, este agente, nunca se arriesgará a un intercambio indirecto que use, ya sea el bien 1 o el bien 4 como medio de cambio, pues nadie querrá ofrecerle algo a cambio de esta última mercancía, i.e., no tendrá acceso al bien 3 que, en última instancia, es su consumo deseado.

En estas dos últimas economías pueden haber intercambios no monetarios, pero para que los agentes se percaten de la alta vendibilidad de algunas mercancías, se requiere de la confrontación de éstos en los intercambios, lo cual no ocurre en las economías minimamente conectadas y más que minimamente conectadas pero menos que plenamente conectadas, por lo que no pueden evolucionar de modo natural hacia un sistema de moneda mercancía. Es sabido que estos dos tipos de economías no pueden sostenerse como un equilibrio en un sistema de intercambio de trueque, pues no se verifica la coincidencia recíproca de las necesidades. Pero ahora se evidencia que éstas tampoco pueden evolucionar de modo natural hacia un sistema de moneda mercancía, aun cuando potencialmente tengan la capacidad de sostenerse como un equilibrio.

Ahora bien, ¿qué ocurre con una economía plenamente conectada? Dado que en la Gráfica anterior se ha supuesto una distribución uniforme de frecuencias habilidad-necesidad, es obvio que para todo agente la estrategia de búsqueda más barata es el truque, pues cada uno de los elementos del conjunto de frecuencias habilidad-necesidad $\left\{e_{i j}\right\}$, coincide con los respectivos elementos del conjunto oferta-demanda $\left\{q_{i j}\right\}$, que el modelo define como el principal determinante de la conducta individual de búsqueda de los agentes, i.e., $\sum_{i} \sum_{j} q_{i j}=1$. Entonces se infiere que solamente cuando la distribución de frecuencias habilidad-necesidad se concentra alrededor de un bien particular, i.e., se verifica la ecuación (2): $\sum_{i} \sum_{j} q_{i j} \leq 1$ y existe oportunidad para una evolución natural de un sistema de intercambio con moneda mercancía, pues la confrontación de los agentes en el intercambio les permite conocer esta estructura asimétrica del conjunto $\left\{e_{i j}\right\}$ y elegir como medio de cambio el bien que posee mayor vendibilidad. 
Por ejemplo, Iwai supone que en la economía plenamente conectada de la Gráfica 1 se tiene $e_{1,2}=e_{2,1}=e_{1,3}=e_{3,1}=e_{1,4}=e_{4,1}=$ $y>e_{2,3}=e_{3,2}=e_{2,4}=e_{4,2}=e_{3,4}=e_{4,3}=x$, es decir que $6 y+6 x=1$. Este es un ejemplo extremo de una economía plenamente conectada con una estructura «real» asimétrica. En este caso, si $2 \log \left(1+\frac{r}{y}\right)<2 \log \left(1+\frac{r}{x}\right)$, esta desigualdad corresponde a la desigualdad ii) del lema 2 . También resulta fácil mostrar que las otras desigualdades de este lema se satisfacen. Ahora, puesto que $2 \log \left(1+\frac{r}{y}\right)<2 \log \left(1+\frac{r}{x}\right) \rightarrow \log \left(1+\frac{r}{y}\right)^{2}<\cdots \log \left(1+\frac{r}{x}\right)$, al desarrollar el binomio cuadrático del lado izquierdo de la desigualdad y considerar una tasa de descuento $r$ pequeña (lo cual implica despreciar los valores cuadráticos de ésta), se tiene que $\log \frac{2 r}{y}<\log \frac{r}{x}$. Puesto que la función logarítmica es creciente, entonces $y>2 x^{20}$.

Es evidente que todo agente de esta economía elegirá el bien 1 como único medio de cambio, excepto por supuesto, aquellos productores y consumidores de 1. Esta economía puede evolucionar a un sistema de mercancía moneda espontáneamente en virtud de sus condiciones «reales» históricamente dadas, pues la vendibilidad y comprabilidad de un bien en particular (1 en este caso), es uniformemente mayor que la de todos los otros bienes. Esto es extremadamente restrictivo para la evolución natural, particularmente en la medida en que la concentración de las frecuencias habilidad-necesidad del bien 1 sean muy desiguales.

La conclusión básica de Iwai es clara: el dinero no necesariamente es un producto natural de la economía humana. Él ha mostrado que la economía plenamente conectada puede evolucionar «naturalmente» hacia un sistema con moneda-mercancía, como Menger lo formula, únicamente cuando su estructura «real», representada por el conjunto $\left\{e_{i j}\right\}$, tiene la configuración

${ }^{20}$ Obsérvese que esta aproximación de la desigualdad es independiente del número $\mathrm{n}$ de bienes. 
«correcta». Esto implica que el origen del dinero debe ser examinado principalmente por consideraciones históricas y no teóricas. En la teoría monetaria de Menger se encuentra un análisis de estas consideraciones históricas ${ }^{21}$.

b) El mecanismo «bootstrap» puede ser interpretado como un modelo formal de la teoría monetaria de Menger

Al endogenizar la regla de Clower, de acuerdo con la cual sólo la moneda compra bienes, emerge el mecanismo «bootstrap», de forma tal que la moneda se sostiene sin ningún soporte real. A tal efecto, Iwai supone arbitrariamente que todos los agentes utilizan uno de los bienes de la economía como único medio de cambio (excepto si son consumidores o productores del mismo). Una vez que éste bien empieza a utilizarse como moneda, emerge una estructura de intercambio totalmente asimétrica en el universo de los bienes, de manera tal que se eleva al máximo la vendibilidad $\left(\mathrm{q}_{\mathrm{im}}\right)$ y la comprabilidad $\left(\mathrm{q}_{\mathrm{mi}}\right)$ de esta mercancía, a costa de la de los otros bienes, pues por la regla de Clower, $\mathrm{m}$ compra bienes y los bienes compran $\mathrm{m}$, pero los bienes no compran bienes.

Esta es la clave para la crítica contra Menger, pues como resultado de que la moneda sea capaz de sostenerse por sí misma, ahora la vendibilidad antes que la causa del dinero, es un efecto, lo cual constituye un «lema anti-Menger». El bootstrap es un mecanismo que borra el origen del dinero; por esta razón Iwai se dirige a la historia para dar una explicación al respecto. «El dinero es dinero porque se usa como dinero», lo cual se traduce en el lema anti-Menger que Iwai refuerza con la siguiente cita:

«One man is king only because other men stand in the relation of subjets to him. They, on the contrary, imagine that they are subjects because he is king» 22 .

${ }^{21}$ Véase la discusión «Sobre el dinero propio de cada pueblo y cada época» en Menger [1871], pp. 233-241.

22 Marx [1867]. 
Pero, en Menger, el dinero también se impone por la práctica y la costumbre. En efecto, hemos visto en la teoría monetaria de Menger la gran trascendencia de la costumbre y la práctica del uso de mercancías con alta capacidad de venta en el origen del dinero. Se advierte que en este autor la vendibilidad es causa del origen de la moneda, a la vez que, la práctica y la costumbre en el uso de una mercancía como moneda, también desempeñan un papel importante en el origen del dinero. Por consiguiente, el lema anti-Menger expresado en el mecanismo bootstrap, bloquea la primera pista que Menger ofrece para el origen de la moneda: la vendibilidad pero, paradójicamente, refuerza la otra pista, a saber: la costumbre y heterogeneidad de los individuos. Entonces, el bootstrap confirma que la práctica y la costumbre juegan un papel fundamental en el origen del dinero. En este sentido, lejos de colapsar la teoría monetaria de Menger, Iwai la fortalece cuando dice que la moneda es moneda porque se usa como moneda.

Así, los agentes que Iwai establece para formular los fundamentales de la economía, $\left\{e_{i j}\right\}$, y el conjunto de frecuencias de oferta y demanda $\left\{q_{i j}\right\}$, son homogéneos en cada zona de intercambio. En cambio, en Menger los individuos no son homogéneos, pues el proceso se inicia con unos agentes que alcanzan grandes beneficios económicos, al aceptar en sus transacciones las mercancías con alta capacidad de venta, a cambio de todos los otros bienes. La práctica y la costumbre de utilizar mercancías con gran capacidad de venta se van haciendo extensivas poco a poco al resto de la sociedad.

\section{El equilibrio monetario de Iwai en el marco del modelo de equilibrio general}

El modelo sin moneda de Iwai establece la estructura de un sistema de mercados organizados, similar al de Walras $\left(\frac{n(n-1)}{2}\right.$ mercados). Esto plantea una relación con la teoría del equilibrio general. En efecto, Iwai considera, como hemos visto, los precios dados exógenamente, y la moneda medio de cambio permite 
que los agentes se confronten en el intercambio, lo cual constituye un avance significativo.

Recordemos que uno de los problemas centrales que trata de resolver Debreu es:

«La explicación de los precios de las mercancías resultantes de la interacción de los agentes de una economía de propiedad privada a través de los mercados» ${ }^{23}$.

Pero en este marco Debreu advierte que:

«No se ofrece... ninguna teoría del dinero y se supone que la economía funciona sin la ayuda de un bien que sirva como medio de cambio...cuando un agente económico se compromete a aceptar la entrega de una cierta cantidad, el producto de esta cantidad por el precio de la mercancía es un número real anotado en el debe de su cuenta...llamado la suma pagada por el agente. Similarmente, un compromiso de entrega resulta en un número real anotado en el haber de su cuenta, y llamado suma pagada al agente. El saldo de su cuenta, es decir el valor neto de todos sus compromisos, guía sus decisiones $»^{24}$.

Por consiguiente, es obvio que se trata de un sistema que supone una organización de los intercambios, o sea, un sistema de cuentas centralizado (una agencia central de compensación) ${ }^{25}$.

«Este es el único sistema coherente con: i) la existencia de $\mathrm{P}^{*}$ como precios de equilibrio general en el sentido completo de la palabra...es evidente que la agencia central de compensación no constituye una solución para... - los intercambios-, sino su negación misma, ya que cada agente nunca se confronta con los demás y sólo entra en relación con la agencia...En este sentido, la organización de las transacciones no es más que una escapatoria» ${ }^{26}$.

\footnotetext{
23 Debreu [1959], p. ix.

24 Ibídem, p. 37, véase también nota 3, p. 47.

25 Véase Benetti [1990], p. 52.

26 Ibídem, pp. 47-48. Benetti hace referencia a que se trata de una escapatoria a las dificultades del truque en los intercambios.
} 
El modelo Arrow-Debreu determina un vector de precios de equilibrio general sin hablar de intercambios. Es un modelo que separa la actividad de intercambio de la determinación de los precios de equilibrio. Los intercambios se efectúan por medio de este sistema de cuentas centralizado a precios de equilibrio. En este sentido, la integración del dinero como medio de cambio como lo concibe Iwai en la teoría del valor del equilibrio gene$\mathrm{ral}^{27}$, elimina el problema de la agencia central de compensación al permitir que los agentes se confronten cuando efectúan sus transacciones.

\section{Emergencia del dinero y los fundamentales de la economía}

Para poder entender la naturaleza del equilibrio monetario de Iwai, se requiere entender cómo se ajustan a través del tiempo las frecuencias de oferta y demanda, $\mathrm{q}_{\mathrm{ij}}$, pues la parte real, las frecuencias habilidad-necesidad, $\mathrm{e}_{\mathrm{i} \mathrm{j}^{\prime}}$, no se alteran. Efectivamente, como lo hemos anticipado al discutir el resultado del mecanismo bootstrap, la solución final de estado estable que se consigna en las ecuaciones (9), (10) y (11), oculta un problema de ajuste. Al elegir el bien $\mathrm{m}$ como medio de cambio universal, $\mathrm{y}$ en virtud de la regla de Clower expresada en (9), ya no es posible el trueque directo. En estas nuevas circunstancias, el número de mercados se reduce a n-1, es decir, aquellas zonas donde convergen los productores de $\mathrm{m}$ y consumidores de los $\mathrm{n}-1$ bienes restantes, con los productores de esos n- 1 bienes y consumidores de $m$.

Pero, bajo estas nuevas condiciones, en estos $n-1$ mercados se presenta una situación de desequilibrio, debido al concurso de nuevos actores que buscan adquirir $\mathrm{m}$, no para consumo, sino como medio de cambio. O sea, en cada una de estas zonas de intercambio, los productores de $\mathrm{m}$ y demandantes de otro bien, están en el lado corto, en el sentido de que se ha incrementado la

27 Habría que resolver la dificultad de adaptar una economía con horizonte infinito a una economía con horizonte finito. 
probabilidad de encontrar con quien intercambiar. En cambio, los productores de un bien en particular, pueden estar demandando m para consumo o como medio de cambio, y están ubicados en el lado largo del mercado, debido a que tendrán menor probabilidad de obtener lo que requieren, en virtud del desequilibrio que esta nueva situación ha provocado.

Obsérvese que en todo este análisis la estructura real permanece intacta, a pesar de que las condiciones de oferta y demanda se han alterado al contraerse el número de mercados. Como veremos a continuación, el ajuste que debe ocurrir en los mercados, para amoldarse a la estructura real de producción y necesidades $\left(\mathrm{e}_{\mathrm{ij}}\right)$, nos da la pauta para afirmar que el equilibrio resultante de este ajuste depende de los fundamentales. Hay que advertir que esta reflexión que hemos hecho respecto al ajuste, es algo que Iwai no toma en consideración.

Aunque resulta difícil resolver y analizar explícitamente un conjunto de intrincadas ecuaciones estocásticas en diferencia, al menos es posible escribir la evolución dinámica de las $\mathrm{q}_{\mathrm{ij}}$ mediante la ley de los grandes números, que se expresa por el producto de frecuencias probabilísticas en las siguientes ecuaciones estocásticas en diferencia:

$$
\begin{aligned}
& \Delta q_{i j}=-q_{m i} q_{i j}+q_{j m}\left(e_{i j}-q_{i j}\right) \quad \forall i, j \neq m \\
& \Delta q_{m j}=-q_{j m} q_{m j}+\sum_{k \neq m} q_{m k} q_{k j} \quad \forall j \neq m \\
& \Delta q_{i m}=0 \equiv q_{i m}=e_{i m} \quad \forall i \neq m
\end{aligned}
$$

La primera ecuación expresa la variación probabilística de la vendibilidad de un bien en términos del bien que se desea adquirir, cuyo intercambio es indirecto, pues primero se vende i a cambio de $m$, y luego se vende m y se compra j. Por esta razón, la comprabilidad que tiene $\mathrm{m}$ del bien $\mathrm{j}, q_{j m}$, multiplica a la diferencia entre la frecuencia habilidad-necesidad, $e_{i j}$, y la frecuencia oferta-demanda $q_{i j}$. A esto se le resta el producto de la comprabilidad que tiene i del bien $\mathrm{m} q_{m i}$ por la frecuencia de oferta-demanda $q_{i j}$.

La segunda ecuación expresa la variación probabilística que tiene la vendibilidad del bien monetizado en términos del bien 
que se desea adquirir, por parte de dos tipos de agentes, a saber: los productores de $\mathrm{m}$ y consumidores de $\mathrm{j}$, así como los productores de $\mathrm{k}$ y consumidores de $\mathrm{j}$ que usan $\mathrm{m}$ como medio de cambio cuando están en la segunda etapa de sus transacciones. Esta variación es igual a la sumatoria del producto de frecuencias comprabilidad que $\mathrm{k}$ tiene de $\mathrm{m}$ por vendibilidad de $\mathrm{k}$ en términos de j. A esta sumatoria se le resta el producto de las frecuencias comprabilidad-vendibilidad que $\mathrm{m}$ tiene de $\mathrm{j}$.

La tercera ecuación expresa la variación probabilística de la comprabilidad del bien monetizado $m$ para los agentes (afortunados) consumidores de $\mathrm{m}$, cuyas transacciones se limitan a una etapa, y por ende la frecuencia oferta de i - demanda de m, coincide con la frecuencia habilidad-necesidad $e_{i{ }^{\prime}}$ razón por la cual esta variación probabilística es nula.

A pesar de la dificultad que tiene la solución explícita de este sistema de ecuaciones estocásticas en diferencia, su solución de estado estable es fácil de formular, haciendo $\Delta q_{i j}=\Delta q_{m j}=\Delta q_{i m}=0$, para luego resolver las ecuaciones simultáneas resultantes, lo cual nos conduce al resultado obtenido en las ecuaciones (9), (10) y (11). El argumento de Iwai es que ese resultado no depende de los fundamentales, $e_{i j}$, dado que todos estos últimos permanecen inalterados a lo largo de este proceso dinámico de intercambio monetario que conduce al equilibrio estacionario.

«...is no real restriction at all, the very process of monetary circulation creates both the general demand and the general supply...A commodity money exchage system is thus capable of sustaining itself as a Nash equilibrium without any 'real' foundation to support it» 28

Nuestra opinión es contraria a la de Iwai, pues consideramos que la estructura real, a pesar de no modificarse, determina el resultado del equilibrio monetario, pues las frecuencias $\mathrm{q}_{\mathrm{ij}}$ tienen que acomodarse a la estructura real. Ese es precisamente el ajuste que desemboca en las ofertas y demandas sociales de $\mathrm{m}$ como medio de cambio.

28 Iwai [1997], p. 27. 


\section{ANEXO}

\section{A.1. Función de probabilidad de Poisson}

Si la variable aleatoria $\mathrm{X}$ se distribuye Binomial: $\mathrm{X} \sim B(n, p)$, entonces, dado que $f(r)=\left(\begin{array}{l}n \\ r\end{array}\right) p^{r}(1-p)^{n-r}$, es difícil calcular $f(r)$ directamente para valores un poco grandes de $r$ y $n$. Muchas veces se prefiere sustituir esta función de densidad por otra de mejor manejo para el cálculo de probabilidades y con un ajuste suficientemente adecuado.

Un caso especial en que esto es posible es para valores pequeños de $p$, tal que el producto $n p$ sea relativamente pequeño aun para valores grandes de $n$. El problema se formula en términos de la búsqueda de un límite para $f(r)$ cuando $p$ tiende a cero, al tiempo que $n$ tiende al infinito, de manera que la esperanza matemática $E(x)=n p$, se mantenga constante e igual a $\lambda$, es decir, $n p=\lambda$.

Evidentemente, se trata de un límite teórico, en virtud de que $p$ tiene un valor fijo en cualquier experimento, por lo que el producto $n p$ crece con el número de veces, $\mathrm{n}$, que se repite el experimento. No obstante, este límite teórico proporciona una buena aproximación a la función de densidad binomial.

$$
\text { Entonces, } \begin{aligned}
& f(r)=\left(\begin{array}{c}
n \\
r
\end{array}\right) p^{r}(1-p)^{n-r}=\left(\begin{array}{l}
n \\
r
\end{array}\right)\left(\frac{\lambda}{n}\right)^{r}\left(1-\frac{\lambda}{n}\right)^{n-r} \\
= & \frac{n(n-1)(n-2) \cdots(n-r+1)}{r !\left(1-\frac{\lambda}{n}\right)^{r}}\left(\frac{\lambda}{n}\right)^{r}\left(1-\frac{\lambda}{n}\right)^{n} \\
= & \frac{\lambda^{r}}{r !} \frac{\left(1-\frac{1}{n}\right)\left(1-\frac{2}{n}\right) \cdots\left(1-\frac{(r-1)}{n}\right)}{\left(1-\frac{\lambda}{n}\right)^{r}}\left(1-\frac{\lambda}{n}\right)^{n} \\
& \operatorname{Lim}\left(1-\frac{1}{n}\right)\left(1-\frac{2}{n}\right) \cdots\left(1-\frac{(r-1)}{n}\right)=1 \\
& n \rightarrow \infty(
\end{aligned}
$$




$$
\operatorname{Lim}_{n \rightarrow \infty}\left(1-\frac{\lambda}{n}\right)^{r}=1
$$

$$
\operatorname{Lim}_{n \rightarrow \infty}\left(1-\frac{\lambda}{n}\right)^{n}=e^{-\lambda} \quad\left(\text { Serie de Mac Laurin } e^{x}=\sum_{n=0}^{\infty} \frac{x^{n}}{n !}\right)
$$

$\therefore f(r)=\frac{e^{-\lambda} \lambda^{r}}{r !}, r=0,1, \ldots$ se llama función de densidad de Poisson.

Se considera que el ajuste de la binomial a la Poisson es bueno cuando $p<0.1$ y $n p<5$. Por esto también se llama función de las pequeñas probabilidades.

Obsérvese que $\sum_{x=0}^{\infty} e^{-\lambda} \frac{\lambda^{x}}{x !}=e^{-\lambda} \sum_{x=0}^{\infty} \frac{\lambda^{x}}{x !}=e^{-\lambda} e^{\lambda}=1$, lo cual demuestra que se trata de una función de densidad.

Esperanza matemática: $E(x)$

$$
\begin{gathered}
E(x)=\sum_{x=0}^{\infty} x \frac{e^{-\lambda} \lambda^{x}}{x !}, \text { puesto que para } x=0, E(x=0)=0, \text { entonces: } \\
E(x)=\sum_{x=1}^{\infty} \lambda \frac{e^{-\lambda} \lambda^{x-1}}{(x-1) !} \text {, definiendo } y=x-1 \\
E(x)=\lambda \sum_{y=0}^{\infty} \frac{e^{-\lambda} \lambda^{y}}{y !}=\lambda
\end{gathered}
$$

Varianza: $E\left(x^{2}\right)-[E(x)]^{2}=\sigma^{2}$

$$
\begin{gathered}
E\left(x^{2}\right)=\sum_{x=0}^{\infty} x^{2} \frac{e^{-\lambda} \lambda^{x}}{x !} \text {, puesto que para } x=0, E\left(x^{2}\right)=0 \text {, entonces: } \\
E\left(x^{2}\right)=\lambda \sum_{x=1}^{\infty} x \frac{e^{-\lambda} \lambda^{x-1}}{(x-1) !} \text {, definiendo } y=x-1 \\
E\left(x^{2}\right)=\lambda \sum_{y=0}^{\infty}(y+1) \frac{e^{-\lambda} \lambda^{y}}{y !}
\end{gathered}
$$




$$
\begin{gathered}
E\left(x^{2}\right)=\lambda\left[\sum_{y=0}^{\infty} y \frac{e^{-\lambda} \lambda^{y}}{y !}+\sum_{y=0}^{\infty} \frac{e^{-\lambda} \lambda^{y}}{y !}\right] \\
E\left(x^{2}\right)=\lambda[\lambda+1]=\lambda^{2}+\lambda \\
\sigma^{2}=\lambda^{2}+\lambda-\lambda^{2}=\lambda
\end{gathered}
$$

La función de densidad Poisson es un caso muy curioso, pues el promedio y la varianza son iguales.

\section{A.2. Distribución de sucesos en el tiempo (procesos de Poisson)}

Supóngase la existencia de un segmento $A$ de longitud $a$ (puede hacerse el mismo análisis con un cuadrado de superficie $a^{2}$ o un cubo de volumen $a^{3}$ ), y otro segmento $B$ de longitud $b$, tal que $B \subset A$. Además, supóngase que la probabilidad de que un punto $x \in A$ también pertenezca a $B$, sea igual a $p=\frac{b}{a}$, independientemente de la posición de $B$ dentro de $A$. Luego, si se dan al azar $n$ puntos en $A$, la probabilidad de que $r$ de ellos estén también en $B$ es una binomial $(r ; n, p):\left(\begin{array}{l}n \\ r\end{array}\right) p^{r}(1-p)^{n-r}=\left(\begin{array}{l}n \\ r\end{array}\right)\left(\frac{b}{a}\right)^{r}\left(1-\frac{b}{a}\right)^{n-r}$. La razón $\frac{n}{a}$ constituye el promedio de puntos, dados al azar, por unidad de longitud. Defínase este promedio $\frac{n}{a}=\lambda$, y asúmase que $a$ crece hasta cubrir toda la recta de los reales, al mismo tiempo que $n$ crece de forma tal que $\lambda$ permanece constante. Por consiguiente, $n p=n \frac{b}{a}=\lambda b$, i.e., se cumple el ajuste de la binomial a la Poisson con parámetro $\lambda b$ :

$$
f(r)=\frac{e^{-\lambda b}(\lambda b)^{x}}{x !}, \quad r=0,1,2, \ldots
$$

Para $r=1$ y $b$ pequeño, defínase $b=\Delta x$, entonces:

$$
f(1)=e^{-\lambda \Delta x} \lambda \Delta x
$$


Expandiendo la serie de Mac Laurin,

$$
e^{-\lambda \Delta x}=\sum_{n=0}^{\infty} \frac{(-\lambda \Delta x)^{n}}{n !}=1-\lambda \Delta x+\frac{(\lambda \Delta x)^{2}}{2 !}-\ldots
$$

y despreciando los términos $(\Delta x)^{2}$ y los que le siguen, se tiene que $f(1)=(1-\lambda \Delta x) \lambda \Delta x=\lambda \Delta x$, expresa que la probabilidad de que un intervalo de longitud contenga uno de los puntos es $\lambda \Delta x$.

El suponer que $p=\frac{b}{a}$, independientemente de la posición de $B$ dentro de $A$, implica que cuando $A$ expresa todo el conjunto de los reales, la probabilidad de que uno de los elementos de este conjunto, dados al azar, pertenezca a $B$ depende únicamente de la longitud de $B$, pero no de la posición de $B$ en $A$. Este supuesto se enuncia diciendo que los elementos están distribuidos en el conjunto $A$ según un proceso de Poisson.

\section{Definición}

Infinitos elementos se distribuyen sobre el conjunto de los reales según un proceso de Poisson, cuando la probabilidad de que uno de ellos pertenezca a un subconjunto $B$ (de longitud $b$ ), condicionado a que pertenezca al subconjunto $A$ (de longitud $a$ ), tal que $B \subset A$, es $\frac{b}{a}$, cualquiera que sea la posición de $A$ en los reales y la posición de $B$ en $A$. Esta definición permite la formulación del siguiente teorema.

\section{Teorema}

Supóngase dados al azar infinitos elementos en los reales según un proceso de Poisson, con un promedio de $\lambda$ elementos por unidad de longitud; la probabilidad de que $r$ de ellos pertenezca a un segmento de longitud $b$ está dada por $f(r)=\frac{e^{-\lambda b}(\lambda b)^{r}}{r !}, \quad r=0,1,2, \ldots$ 
Ahora, $\lambda$ puede ser considerado el tiempo promedio en el cual se manifiesta un fenómeno, y b un intervalo de tiempo particular, dentro del cual se desea establecer la probabilidad de que dicho fenómeno ocurra un número, r, determinado de veces.

Considérese ahora un punto cualquiera $k$ sobre la recta de los reales. ¿Cuál es la probabilidad de que el $r$-ésimo punto, a partir de $k$, de un proceso de Poisson se localice a una distancia comprendida entre $x$ y $x+\Delta x$ de $k$ ? De acuerdo con le teorema anterior, la probabilidad de que en el segmento de longitud $x$ existan $r-1$ puntos es: $f_{r}(x)=\frac{e^{-\lambda x}(\lambda x)^{r-1}}{(r-1) !}$ y la probabilidad de que en el segmento $\Delta x$ exista un punto, es $\lambda \Delta x$, según se estableció para $f(1)$. Entonces, por independencia, la probabilidad buscada es igual al producto de estos dos: $f_{r}(x) \Delta x=\frac{\lambda^{r}}{(r-1) !} e^{-\lambda x} x^{r-1}$

De aquí se deduce que la distancia media entre un punto cualquiera y el $r$-ésimo punto del proceso es:

$$
E(x)=\int_{0}^{\infty} x f_{r}(x) d x=\frac{\lambda^{r}}{(r-1) !} \int_{0}^{\infty} e^{-\lambda x} x^{r} d x
$$

Sea $\lambda x=t$, entonces $d x=\frac{1}{\lambda} d t$, y se tiene:

$$
\begin{gathered}
E(x)=\frac{\lambda^{r}}{(r-1) !} \int_{0}^{\infty} e^{-t}\left(\frac{t}{\lambda}\right)^{r} \frac{d t}{\lambda} \\
E(x)=\frac{1}{(r-1) ! \lambda} \int_{0}^{\infty} e^{-t} t^{r} d t
\end{gathered}
$$

Donde $\int_{0}^{\infty} e^{-t} t^{r} d t$ define la función matemática Gamma $\Gamma_{(r)}=\int_{0}^{\infty} e^{-t} t^{r-1} d t \therefore \Gamma_{(r+1)}=\int_{0}^{\infty} e^{-t} t^{r} d t$. Al integrar por partes, esta función se vuelve recursiva y es igual a $r(r-1)(r-2) \ldots(3)(2)(1)=r$ !

Por consiguiente, $E(x)=\frac{r}{\lambda}$. Para el caso particular en que $r=1$, se tiene: $E(x)=\frac{1}{\lambda}$. 


\section{REFERENCIAS BIBLIOGRÁFICAS}

Benetti, C., [1990], Moneda y Teoría del Valor, México, Fondo de Cultura Económica/Economía Contemporánea.

Debreu, G., [1959], Teoría del Valor, un Análisis Axiomático del Equilibrio General, Barcelona, Bosch Casa Editorial, 1973, traducción al español.

IWAI, K., [1988미, Evolution of Money - A Search-Theoretic Foundation of Monetary Economics, CARESS Working Paper, \#883, Universidad de Pensilvania y Universidad de Tokio.

- [1988b], Fiat Money and Agreagate Demand Management in a Search Model of Decentralized Exchanges, CARESS Working Paper, \#88-16, Universidad de Pensilvania, Universidad de Princeton y Universidad de Tokio.

- [1997], Evolution of Money, (First Draft), Workshop on «Evolution Economics», Universidad de Tokio y Universidad de Siena.

Menger, C., [1871], Principios de Economía Política, Barcelona, Unión Editorial S.A., edición en español, 1996. Traducción del alemán: Marciano Villanueva.

- (1892), «On the Origen of Money». Economic Journal, vol. 2, pp. 239-55. Traducción de C.A. Foley.

SANTALÓ, L.A., [1970], Probabilidad e Inferencia Estadística, Washington, Organización de Estados Americanos. 